\title{
First issue of EAHT
}

From volume 34, issue 3, the journal title is changed to "Environmental Analysis, Health and Toxicology (EAHT)". With successful progress of EHT, the Korean society of environmental health and toxicology (KOSEHT) and Korea society for environmental analysis (KSFEA) agreed to publish a periodical journal together, extending the scope of EHT. As described in the revised Instruction to Authors, EAHT invites articles, letters, reviews, etc. in a broad area of environmental studies related to chemicals in the various environmental media and related effects on human and ecosystem health. Major areas of interests include but not limited to biological and chemical analysis for environment, environmental chemistry and exposure to chemicals, ecotoxicology and ecological risks, human toxicology, risk assessment, environmental health and policy.
To cover broader scope of the journal, we also strengthened the editorial board inviting experts from various geographic regions. The most important role of an academic journal is to provide a room for exchanging new ideas and reporting novel scientific findings toward environmental welfare.

"An expert is a person who has made all the mistakes that can be made in a very narrow field." - Niels Bohr (1885-1962)

Jung-Hwan Kwon http://orcid.org/0000-0002-6341-7562

Editor-in-Chief Environmental Analysis Health and Toxicology Division of Environmental Science and Ecological Engineering, Korea University, Seoul, Korea 